\title{
MOLECULAR STUDIES OF HERBIG-HARO OBJECTS
}

\author{
Salvador Curiel \\ Harvard-Smithsonian \\ Center for Astrophysics \\ 60 Garden Street (MS 10) \\ Cambridge, MA 02138
}

\begin{abstract}
Recent ultraviolet, infrared, millimeter and centimeter-wavelength observations have revealed that $\mathrm{HH}$ objects are also sources of molecular line emission. Infrared observations have shown that $\mathrm{H}_{2}$ line emission in $\mathrm{HH}$ objects presents a wide and complex variety of morphologies, being in most of the cases similar to that observed at optical wavelengths. New high-angular resolution radio observations of molecular lines have revealed that small high-density condensations are frequently found in association with $\mathrm{HH}$ objects. Below, a brief review of molecular emission in $\mathrm{HH}$ objects is presented.
\end{abstract}

\section{$\mathrm{H}_{2}$ EMISSION ASSOCIATED WITH HH OBJECTS}

The near-infrared spectral region contains a number of emission lines of molecular hydrogen, which have been proven to be important diagnostics for the molecular component of the gas in $\mathrm{HH}$ objects. The strongest and most commonly studied infrared line is the $\mathrm{v}=1-0 \mathrm{~S}(1) \mathrm{H}_{2}$ line at $2.12 \mu \mathrm{m}$. This line was first detected and mapped in $\mathrm{HH}$ objects using single beam techniques, and recently, using twodimensional array detectors. $\mathrm{H}_{2}$ emission has been searched and detected in many Herbig-Haro objects, including HH1-2 (Harvey et al.1986; Hartigan et al.1991), $\mathrm{HH}$ 7-11 (Zealey et al.1984; Lightfoot and Glencross 1986; Hartigan et al.1989; Burton et al.1989; Garden et al.1990; Stapelfeldt et al.1991), HH32 (Zealey et al.1986), HH43 (Schwartz et al.1988b), HH52/53/54 (Sandell et al.1987), HH 6 and $\mathrm{HH}$ 12 (Lane and Bally 1986; Stapelfeldt et al.1991), Cep A/GGD 37 (Bally, and Lane 1991), and several additional sources in surveys by Elias (1980), Schwartz et al.(1987), and Wilking et al.(1990); see also the review by Lane (1989). Highresolution infrared spectra of $\mathrm{H}_{2}$ lines, from several $\mathrm{HH}$ objects, have been obtained by Doyon and Nadeau (1988), Zinnecker et al.(1989), Brand et al.(1989) and Carr (1990). A general result of these observations is that $\mathrm{H}_{2}$ lines are much more narrow than optical lines. In particular, the width of the $\mathrm{S}(1)$ line is typically only half of the width of the $\mathrm{H} \alpha$ line in the same objects, and the observed central velocities of the $\mathrm{H}_{2}$ lines are also lower than those of the optical lines (Zinnecker et al.1989).

The vibrational and rotational transitions of the $\mathrm{H}_{2}$ molecule can be excited either by shocks (via collisional excitation in gas heated by the shocks to a few thousand degrees) or by absorption of ultraviolet radiation (in the Lyman and Werner bands with subsequent cascade to lower levels). Because the line strengths 
produced by the collisional and the fluorescent processes are significantly different, it is possible to distinguish between these mechanisms by comparing the line strength of lines from different levels such as the $v=1-0$ and $v=2-1 S(1)$ transitions at 2.122 $\mu \mathrm{m}$ and $2.247 \mu \mathrm{m}$, respectively. A general description of the characteristics expected for $\mathrm{H}_{2}$ emission excited in several different situations is given by Wolfire and Königl (1991). Numerical simulations predict that the expected line ratio of these lines from shocked gas is of the order of 10, whereas in photo excited gas it is $\sim 2$ (e.g., Black and van Dishoeck 1987). Molecular line ratios measured in several $\mathrm{HH}$ objects are 10 (e.g., Schwarts et al.1987, 1988a), which are consistent with the predictions of nondissociative, low velocity shock wave models (e.g., Shull and Hollenbach 1978). In general, the $\mathrm{H}_{2}$ emission seems to trace low velocity shock waves (with velocities around 10 to $40 \mathrm{~km} \mathrm{~s}^{-1}$ ), while the optical emission traces much faster shocks (with typical velocities of about $100 \mathrm{~km} \mathrm{~s}^{-1}$, or more). However, there is some evidence that other molecular excitation mechanisms may be at work in some of the objects. For instance, ultraviolet lines identified as $\mathrm{H}_{2}$ Lyman band emission have been observed in some low-excitation $\mathrm{HH}$ objects such as $\mathrm{HH} 43$ and HH 47 (Schwartz 1983). Likewise, the UV continuum that has been detected in several $\mathrm{HH}$ objects (such as $\mathrm{HH} 1$ and 2) and previously attributed to atomic hydrogen two-photon emission (e.g., Dopita et al.1982; Brugel et al.1982) may arise, at least in part, from $\mathrm{H}_{2}$ photodissociation (Böhm et al.1987). A detailed review of UV observation of $\mathrm{HH}$ objects has been recently presented by Brugel (1989).

\section{COMPARISON OF OPTICAL AND IR EMISSION}

Since $\mathrm{H}_{2}$ lines and optical lines trace different components of the shocked gas, a comparison of the spatial distribution of optical and infrared emission in $\mathrm{HH}$ objects may provide essential clues to their flow structure. With the new infrared array detectors, it is now possible to study the $\mathrm{H}_{2}$ emission distribution at resolutions comparable to optical images. Such studies have been performed by Schwartz et al.(1988), Hartigan et al.(1989), Garden et al.(1990), Stapelfeldt et al.(1991), and Lane et al.(1991). The technique and new results are discussed in a recent review by Lane (1989). These studies have shown that the $\mathrm{H}_{2}$ line emission in $\mathrm{HH}$ objects presents a wide and complex variety of morphologies. Although the overall distribution of the $\mathrm{H}_{2}$ emission is similar to that observed at optical wavelengths, they have subtle but important differences. To illustrate these differences, a general description of the morphology of three regions is presented; $\mathrm{HH} \mathrm{1-2,} \mathrm{Cepheus} \mathrm{A}$, and $\mathrm{HH} 7-11$.

a) HH1-2. Large proper motions and wide optical lines have been observed in this region indicating that high velocity (up to $\sim 200 \mathrm{~km} \mathrm{~s}^{-1}$; Hartmann and Raymond 1984) shock waves are taking place. However, $\mathrm{H}_{2}$ line profiles exhibit narrow linewidths (of about $40 \mathrm{~km} \mathrm{~s}^{-1}$; Zinnecker et al.1989), suggesting that $\mathrm{H}_{2}$ molecules are excited by weaker shock waves. Although $\mathrm{HH} 1$ and HH2 share a common energy source, they exhibit different morphologies at both, infrared and optical wavelengths (see Figure 1). HH1 is a clear example of a well defined bow shaped optical object where the $\mathrm{H}_{2}$ lines are emitted from the wings (away from the apex) of the bow shock. This morphology is consistent with a jet model (or a bullet model) with a bow shock at its end. In this model, the external $\mathrm{H}_{2}$ molecules entering the bow shock will be dissociated near its apex (where the shock velocity is generally greater than $100 \mathrm{~km} \mathrm{~s}^{-1}$ ) and $\mathrm{H}_{2}$ line emission will therefore predominantly arise from the wings of the bow shock (where the 

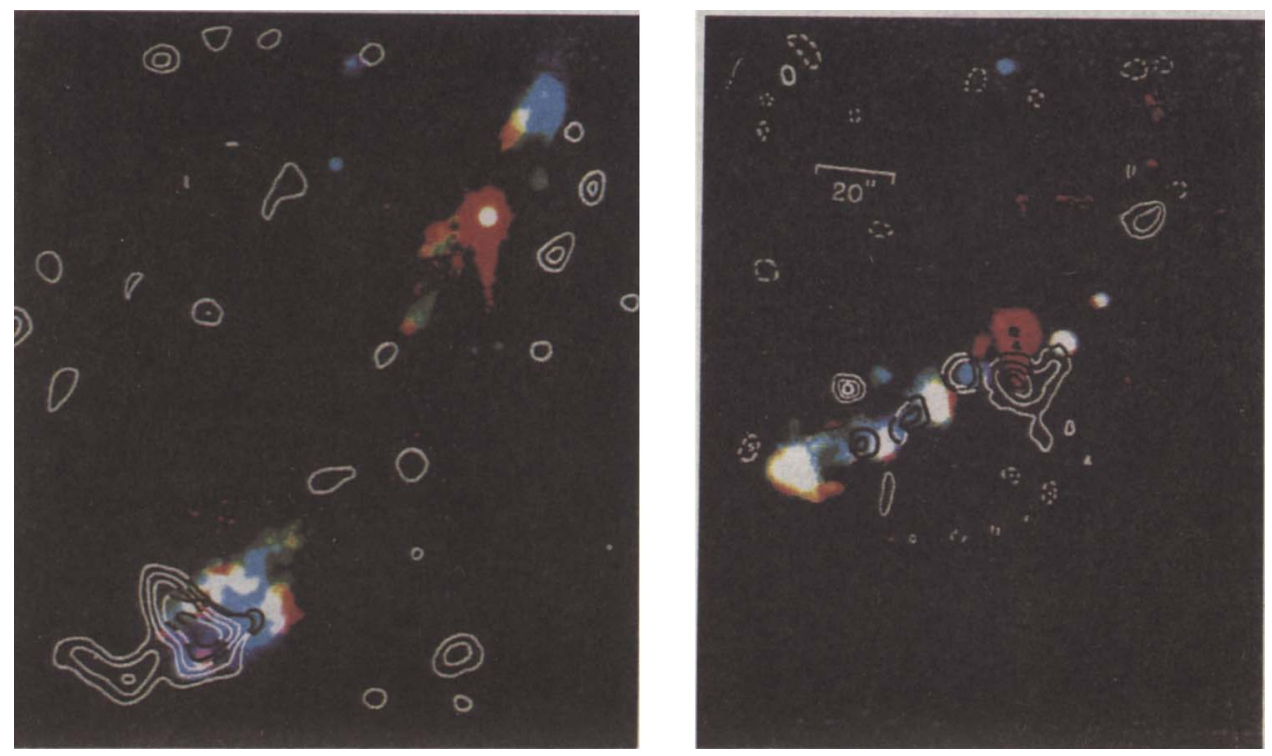

Figure 1. Comparison of an infrared $\mathrm{H}_{2}$ line image with optical $\mathrm{H}_{\alpha}$ and [SII] images of the HH1-2 (left) and HH7-11 (rigth) regions obtained by Hartigan et al.(1991) and Hartigan et al.(1989). This figure also shows the spatial distribution of $\mathrm{NH}_{3}$ in $\mathrm{HH} 1-2$ and $\mathrm{HCO}^{+}$in $\mathrm{HH} 7-11$ with respect to the optical and infrared emission.
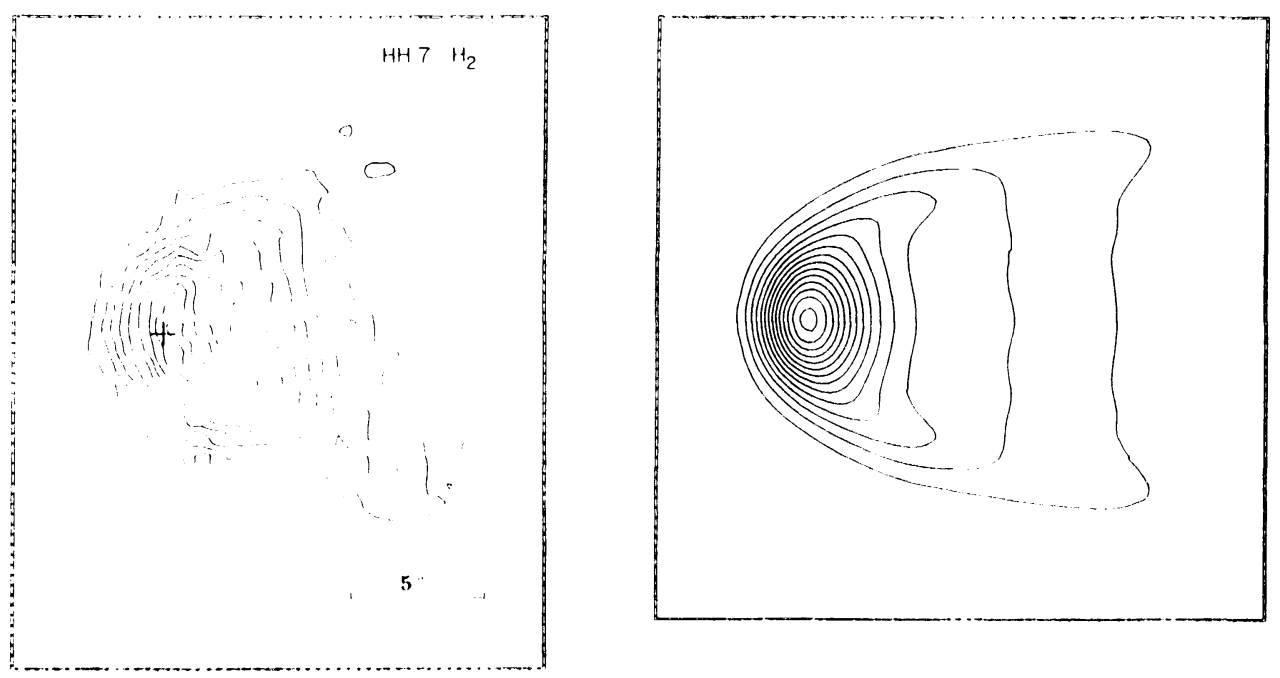

Figure 2. Comparison of the integrated 1-0S(1) $\mathrm{H}_{2}$ flux map of $\mathrm{HH} 7$ (left) with that calculated for a bow-shaped shock wave with a magnetic precursor (rigth), using a shock velocity of $100 \mathrm{~km} \mathrm{~s}^{-1}$, and an inclination angle of $40^{\circ}$ with respect to the line-of-sight. 
shocks are oblique and thus, weaker), having small radial velocities (compared with optical lines). The fact that the $\mathrm{H}_{2}$ emission is observed mainly along the edge of one side of the optical object may be due to a geometrical projection (being favored the regions in the wings of the shock wave where, viewed from an angle, the molecular column density along the line-of-sight is greater) or to inhomogeneities in the ambient gas (e.g., if the flow is hitting the edge of a molecular condensation such as that detected in this region by Davis et al.[1990]). On the other hand, HH 2 has a more complicated optical structure, with several knots and extended emission connecting them. A similar morphology is observed at infrared wavelengths but with less evident extended emission. When infrared images are superimposed to optical images (see Figure 1), the $\mathrm{H}_{2}$ emission seems to be produced predominantly upstream with respect to the $\mathrm{H}_{\alpha}$ and [SII] lines (i.e., toward the energy source of the system). However, most of the optical knots seem to have an $\mathrm{H}_{2}$ counterpart, suggesting a common origin for the $\mathrm{H}_{2}$ and optical lines.

b) Cepheus A/GGD 37. A particularly intriguing case is the Cepheus A region (e.g., Hartigan et al.1986, Lenzen 1988), which has been mapped at arcsecond resolution (Lane 1989, Bally and Lane 1991). This $\mathrm{HH}$ object presents large proper motions $\left(110-250 \mathrm{~km} \mathrm{~s}^{-1}\right)$ and extremely broad optical lines indicating shock velocities up to almost $500 \mathrm{~km} \mathrm{~s}^{-1}$. On the other hand, the $\mathrm{H}_{2}$ lines are much narrower with linewidths of $25-45 \mathrm{~km} \mathrm{~s}^{-1}$ (Doyond and Nadeau 1988), suggesting that the $\mathrm{H}_{2}$ molecules are excited by much less energetic shock waves. The GGD $37 \mathrm{HH}$ object has an overall bow shock morphology, with complex bow shaped substructures (Bally and Lane 1991; Lane et al.1991). A similar morphology is seen in molecular hydrogen, but in this case the arcs are softer and less protruding. As in the case of $\mathrm{HH} 1$, it appears that the $\mathrm{H}_{2}$ lines are emitted mainly from the wings of the bow shocks, where the shocks are oblique and thus much weaker (Bally and Lane 1991). The morphology and spectroscopic characteristics of this object seem to be consistent with a model of a bow shock formed around an interstellar "bullet" moving through a molecular medium and viewed from an angle (Hartigan et al.1986; Lane 1989; Bally and Lane 1991).

c) HH7-11. HH7-11 is a nearly collinear system of optical emission knots in the L1450 molecular cloud near NGC 1333 (see Strom et al.1986). These objects have optical radial velocities $\left(-200\right.$ to $\left.-40 \mathrm{~km} \mathrm{~s}^{-1}\right)$ and line profiles indicative of shock velocities $\sim 100 \mathrm{~km} \mathrm{~s}^{-1}$ (Solf and Böhm 1987), but the spectra show strong [OI] and [SII] lines characteristic of a low-velocity $\left(\sim 30 \mathrm{~km} \mathrm{~s}^{-1}\right)$ shock. The $\mathrm{H}_{2} 1-0 \mathrm{~S}(1)$ emission line is centered at much lower radial velocities of -60 to $0 \mathrm{~km} \mathrm{~s}^{-1}$ (Zinnecker et al.1989), an important fact to shock models of the region. Intense $\mathrm{H}_{2}$ emission is observed in three of the $\mathrm{HH}$ objects ( $\mathrm{HH} 7, \mathrm{HH} 8$, and $\mathrm{HH} 10$ ), while weak emission is observed in $\mathrm{HH} 9$ and no emission is associated with $\mathrm{HH} 11$ (Hartigan et al.1989; Figure 1). HH7 is a particularly interesting object which has a bow-shaped morphology at both, optical and infrared-wavelengths, with the $\mathrm{H}_{2}$ and [SII] images coinciding within an arcsecond. Recent results indicate that in the case of $\mathrm{HH} 7$ the $\mathrm{H}_{2}$ emission seems to arise from a "magnetic precursor" in front of a J-shock (Hartigan et al.1989; Carr 1990; and Stapelfeldt et al.1991). In HH8 and $\mathrm{HH} 10$, the $\mathrm{H}_{2}$ emission seems to be produced upstream with respect to the $\mathrm{H}_{\boldsymbol{\alpha}}$ and [SII] lines (i.e., toward the energy source of the system). In these two objects, the spatial and velocity characteristics of the emitting gas reveal a geometry more complex than that of a simple bow shock or planar shock. HH11 remains an enigma, and could represent a second ejection from SVS 13 (the presumed energy source of the system). The low-excitation spectrum observed in this object is particularly 
difficult to explain since $\mathrm{HH} 11$ has no visible $\mathrm{H}_{2}$ emission.

Recently, detailed $\mathrm{H}_{2}$ velocity mapping with spatial resolution of the order of $1^{\prime \prime}$ has been obtained combining two-dimensional infrared detector arrays, such as IRCAM at UKIRT, with Fabry-Perot spectrophotometers (e.g., Carr 1990). This new type of observations will be very useful to establish the shock type and geometry of individual $\mathrm{HH}$ objects and optical jets. Likewise, further information can be obtained by calculating infrared line intensities and profiles from bow-shaped shock waves with magnetic precursors, similar to those calculated for optical lines by Hartigan et al.(1987). This type of model has been used by Carr (1990) and Curiel (1991) to calculate the $\mathrm{H}_{2}$ 1-0S(1) line profile for a bow shock geometry, for which they obtained a profile very similar to the spatially integrated line profile for $\mathrm{HH}$. Figure 2 shows a comparison of an integrated $\mathrm{H}_{2}$ flux map obtained for $\mathrm{HH} 7$ by Hartigan et al.(1989) and those calculated for a bow-shaped shock wave with a magnetic precursor, using a shock velocity of $100 \mathrm{~km} \mathrm{~s}^{-1}$, and an inclination angle of $40^{\circ}$ with respect to the line-of-sight (Curiel 1991). The adopted shock is a very simplified jet model in which both the temperature and velocity increase linearly from an initial value to their maximum values in the precursor. It is important to notice how the global morphology predicted by this simple model resemble that observed. This type of shock wave seems to be a very promising way to explain the spatial coincidence of optical and infrared emission, and the line profiles observed in $\mathrm{HH} 7$ and other $\mathrm{HH}$ objects.

\section{$\mathrm{HCO}^{+}$AND $\mathrm{NH}_{3}$ EMISSION ASSOCIATED WITH HH OBJECTS}

Recent high-angular resolution observations of molecular lines, carried out with the VLA, Hat Creek, NMA and JCMT radio telescopes, have shown that small high-density condensations are frequently found at the border of $\mathrm{HH}$ objects and aligned with the outflow direction. The most commonly studied lines are those of $\mathrm{HCO}^{+}(\mathrm{J}=1 \rightarrow 0)$ and $(\mathrm{J}=3 \rightarrow 2)$ at millimeter wavelengths, and $\mathrm{NH}_{3}(1,1)$ and $(2,2)$ at centimeter wavelengths. $\mathrm{HCO}^{+}$clumps have been detected in association with HH 7-11 (Rudolph and Welch 1988; Figure 1), and with HH 1-2 (Davis et al.1990). Ammonia condensations have been observed toward HH 25-26 (Torrelles et al.1989), and HH 2 (Torrelles et al.1991, private communication; Figure 1); see also the review by Torrelles (1990). These $\mathrm{NH}_{3}$ and $\mathrm{HCO}^{+}$condensations or clumps have typical densities of $\mathrm{n}\left(\mathrm{H}_{2}\right) \simeq 10^{5} \mathrm{~cm}^{-3}$, radial velocities similar to those of the ambient molecular cloud and typical sizes of a few arcseconds. Although the number of reported cases is still comparatively small, the high detection rate suggests that this emission may be a common feature of $\mathrm{HH}$ objects.

The association of $\mathrm{HH}$ objects with molecular condensations suggests that at least these $\mathrm{HH}$ objects could be ambient dense gas shocked by a wind. If this is the case, the observed high-density clumps associated with the $\mathrm{HH}$ objects would be ambient cloudlets (perhaps uniformly distributed in the molecular cloud) that have been compressed by a shock wave, enhancing the density by at least a factor of $\sim 100$ in order to be detectable with molecular tracers of high-density gas such as $\mathrm{NH}_{3}$ and $\mathrm{HCO}^{+}$(e.g., Rudolph and Welch 1988, and Torrelles 1990). However, the compression of the ambient molecular gas would also imply an acceleration of the molecular gas to velocities different to the ambient molecular cloud velocity. At present, such acceleration has not been observed in the high-density condensations associated with $\mathrm{HH}$ objects. The molecular lines observed typically have radial velocities of only $\sim 1 \mathrm{~km} \mathrm{~s}^{-1}$ and widths of $\leq 1 \mathrm{~km} \mathrm{~s}^{-1}$. Another possible 
explanation to this association is that the uv radiation emitted by the shock waves excite and heat ambient high-density cloudlets nearby. In this case, the cloudlets would have radial velocities similar to that of the molecular cloud velocity, and line widths given by thermal broadening. However, calculations will be needed to establish if the radiation emitted by shock waves is capable of exciting $\mathrm{HCO}^{+}$and $\mathrm{NH}_{3}$ in molecular condensations to the observed levels.

-I am grateful to A.P. Lane and B. Reipurth who kindly provided some excelent images which I used during the presentation of this work. I am also grateful to J.M. Torrelles for comments on an early version of this manuscript and for providing the unpublished $\mathrm{NH}_{3}$ contour map used in Fig. 1 .

\section{REFERENCES}

Bally, J., and Lane, A.P. 1991, in Astrophysics with Infrared arrays, ASP vol. 14, ed. R. Elston, p. $273-278$.

Black, J.H., and van Dishoeck 1987, Ap. J., 322, 412.

Böhm, K., Bührke, Th., Raga, A., Brugel, E., Witt, A., and Mundt, R. 1987, Ap. J., 316, 349.

Brand, P.W.J.L., Toner, M.P., Geballe, T.R., and Webster, A.S. 1989, MNRAS, 237, 1009.

Brugel, E.W. 1989, in ESO-Workshop on Low Mass Star Formation and Pre-Main Sequence Objects, ed. Bo Reipurth, p. 311-329.

Bugel, E.W., Shull, J.M., and Seab, C.G. 1982, Ap. J., 262, L35.

Burton, M.G., Brand, P.W.J.L., Geballe, T.R., and Webster, A.S. 1989, MNRAS, 236, 409.

Carr, J.S. 1990, Bull. AAS, 21, 1086.

Curiel, S. 1991, PhD Thesis, Universidad Nacional Autónoma de México.

Davis, C.J., Dent, W.R.F., and Bell Burnell, S.J. 1990, MNRAS, 244, 173.

Dopita, M.A., Binette, L., and Schwartz, R.D. 1982, Ap. J., 261, 183.

Doyon, R., and Nadeau, D. 1988, Ap. J., 334, 883.

Elias, J.H. 1980, Ap. J., 241, 728.

Garden, R.P., Russell, A.P.G., and Burton, M.G. 1990; Ap. J., 354, 232.

Hartigan, P., Curiel, S., and Raymond, J. 1989, Ap. J. (Letters), 347, L31.

Hartigan, P., Curiel, S., and Raymond, J. 1991, in preparation.

Hartigan, P., Lada, C.J., Stocke, J., and Tapia, S. 1986, A. J., 92, 1155.

Hartigan, P., Raymond, J., and Hartmann, L. 1987, Ap. J., 316, 323.

Hartmann, L., and Raymond, J. 1984, Ap. J., 276, 560.

Harvey, P.M., Joy, M., Lester, D.F., and Wilking, B.A. 1986, Ap. J., 301, 346.

Lane, A.P. 1989, in ESO-Workshop on Low Mass Star Formation and Pre-Main Sequence Objects, ed. Bo Reipurth, p. 331-348.

Lane, A.P., and Bally, J. 1986, Ap. J., 310, 820.

Lane, A.P., Bally, J., and Hartigan, P. 1991, in preparation.

Lenzen, R. 1988, Astr. Ap., 190, 269.

Lightfoot, J.F., and Glencross, W.M. 1986, MNRAS, 221, 993.

Rudolph, A., and Welch, W.J. 1988, Ap. J. (Letters), 326, L31.

Sandell, G., Zealey, W.J., Williams, P.M., Taylor, K., and Strey, J.V. 1987, Astr. Ap., 182, 237.

Schwartz, R.D. 1983, Ann. Rev. Astr. Ap., 21, 209.

Schwartz, R.D., Cohen, M., and Williams, P.M. 1987, Ap. J., 322, 403.

Schwartz, R.D., Cohen, M., and Williams, P.M. 1988a, Ap. J., 333, 1035.

Schwartz, R.D., Williams; P.M., Cohen, M., and Jennings, D.G. 1988b, Ap. J. (Letters), 334, L99.

Shull, J.M., and Hollenbach, D.J. 1978, Ap. J., 220, 525.

Solf, J., and Böhm, K.H. 1987, A. J., 93, 1172 .

Stapelfeldt, K.R., Beichman, C.A., Hester, J.J., Scoville, N.Z., and Gautier III, T.N. 1991, Ap. J. (Letters), , in press.

Strom, K.M., Strom, S.E., Wolff, S.C., Morgan, J., and Wenz, M. 1986, Ap. J. Suppl., 62, 39.

Torrelles, J.M. 1990, in Atoms, Ions, and Molecules: New Results in Spectral Line Astrophysics, ASP vol. 16, eds. A.D. Haschick and P.T.P. Ho, p. 257-268.

Torrelles, J.M., Ho, P.T.P., Rodríguez, L.F., Cantó, J., and Verdes, L. 1989, Ap. J., 346, 756.

Wilking, B.A., Schwartz, R.D., Mundy, L.G., and Schultz, A.S.B. 1990, A. J., 99, 344.

Wolfire, M.G., and Königl, A. 1991, Ap. J., in press.

Zealey, W.J., Williams, P.M., and Sandell, G. 1984, Astr. Ap., 140, L31.

Zealey, W.J., Williams, P.M., Taylor, K.N., Storey, J.W., and Sandell, G. 1986, Astr. Ap., 158, L9.

Zinnecker, H., Mundt, R., Geballe, T.R., and Zealey, W.J. 1989, Ap. J., 342, 337. 